\title{
Ruxolitinib In The Treatment Of Polycythemia Vera: An Update On Health-Related Quality Of Life And Patient-Reported Outcomes
}

This article was published in the following Dove Press journal:

Journal of Blood Medicine

\author{
Shashank Cingam (I) \\ Lainey Flatow-Trujillo ${ }^{2}$ \\ Leslie A Andritsos' \\ Cecilia Arana $\mathrm{Yi}\left(\mathbb{D}^{\prime}\right.$ \\ 'University of New Mexico \\ Comprehensive Cancer Center, \\ Albuquerque, NM, USA; ${ }^{2}$ Department of \\ Internal Medicine, University of New \\ Mexico School of Medicine, Albuquerque, \\ NM, USA
}

Correspondence: Cecilia Arana Yi University of New Mexico

Comprehensive Cancer Center, I20I

Camino De Salud NE, Albuquerque, NM

87I3I, USA

Email caranayi@salud.unm.edu

\begin{abstract}
Polycythemia vera (PV) is a rare myeloproliferative neoplasm (MPN) associated with significant impairment in quality of life (QoL) due to disease-related symptoms and complications. Assessment of disease burden constitutes standard monitoring of symptoms and response. Conventional treatments for MPN, such as hydroxyurea, phlebotomy, or interferon, have not shown a significant impact in QoL or patient-reported outcomes (PRO). Ruxolitinib (RUX) is a JAK2 inhibitor approved for patients intolerant or resistant to hydroxyurea (HA). We conducted a systematic review of clinical trials of RUX in patients with PV that incorporated PRO measures to evaluate the effects on PRO and QoL. Three randomized Phase 3 studies reported in four publications were relevant for analysis. Although the small number of trials and potential for treatment bias in the review, treatment with RUX was associated with improved QoL and PRO in PV patients intolerant or resistant to hydroxyurea.
\end{abstract}

Keywords: polycythemia vera, ruxolitinib, quality of life, patient-reported outcomes

\section{Introduction}

Polycythemia vera (PV) is a rare myeloproliferative neoplasm (MPN) characterized by increased red blood cell mass, bone marrow panmyelosis, and Janus kinase 2 (JAK2) mutation. ${ }^{1,2}$ Constitutional symptoms such as fatigue, weight loss, night sweats and pruritus (so-called "cytokine symptoms") are present in approximately $61 \%$ of patients with PV and affect the quality of life (QoL), overall health and productivity. $^{3-6}$ Healthcare utilization and costs are also higher in patients with PV than in non-cancer controls. ${ }^{7}$

Patient-reported outcomes (PRO) measures are tools for the objective evaluation of symptoms, physical and social functioning, and mental health and well-being. ${ }^{8}$ Despite their pivotal importance in the understanding of disease- and treatmentrelated adverse events, information regarding PRO in hematologic malignancies remains sparse. ${ }^{9}$ However, growing recognition of the symptom burden in PV has led to the incorporation of PRO instruments for symptom assessment into the standard evaluation of MPN's (NCCN guidelines, version 2.2019).

In clinical trial settings, PRO instruments evaluate therapeutic responses, quality of life, and prognosis. ${ }^{10}$

The goals of PV therapy are to decrease thrombotic events, manage symptoms, minimize adverse effects of treatment, and reduce the risk of transformation to myelofibrosis or leukemia. 1,11,12 Treatments for PV include phlebotomy, aspirin, 
and cytoreductive therapies such as hydroxyurea (HA), anagrelide, and interferon. HA decreases the hematocrit, reduces the spleen size, and lowers thrombotic risk, but does not reduce symptom burden in all patients. ${ }^{13,14}$ Intolerance of or resistance to HA occurs in approximately $25 \%$ of patients and is associated with poor outcomes. ${ }^{15}$

Ruxolitinib (RUX) is an oral JAK1/JAK2 inhibitor approved for the management of myelofibrosis and for PV patients who are intolerant or resistant to HA, per modified ELN criteria. ${ }^{4,16,17}$ In myelofibrosis, treatment with RUX may result in the reduction of spleen size, decreased symptom burden, and improvement in QoL measures. ${ }^{18,19}$ In PV, the phase 3 RESPONSE study demonstrated the superiority of RUX compared to the best available therapy in the reduction of hematocrit, spleen size, and PV symptoms. ${ }^{4}$ Since then, additional studies have explored the impact of RUX on symptom burden and QoL. Herein we present a review of the PRO literature in PV patients treated with RUX and propose future concepts for research.

\section{Methods}

\section{Search Criteria}

We conducted a systematic review in PubMed, Medline, EMBASE, and Cochrane of English-language scientific articles using the MeSH-terms "randomized clinical trials" "polycythemia vera" "ruxolitinib," "quality of life" and "patient-reported outcome" as title or abstract terms from January 2000 to January 2018. We reviewed the bibliographies of all retrieved papers to identify randomized controlled trials that studied the effect of RUX on symptoms and quality of life as primary or secondary endpoints. The exclusion criteria were the following:

- Non-English publication

- Publication before 2000

- Patients are younger than 18 years.

- Non- PV myeloproliferative neoplasms.

- Only abstract available.

We synthesized the data according to PRISMA guidelines. (Figure 1). ${ }^{20}$ The quality of the study was assessed using the Mixed Methods Appraisal Tool (MMAT). ${ }^{21}$

\section{Data Extraction And Analysis}

We extracted the following data: Demographics, number of patients enrolled, symptoms, adverse effects, missing data and results, and instruments of QoL and PRO assessments. (Table 1)

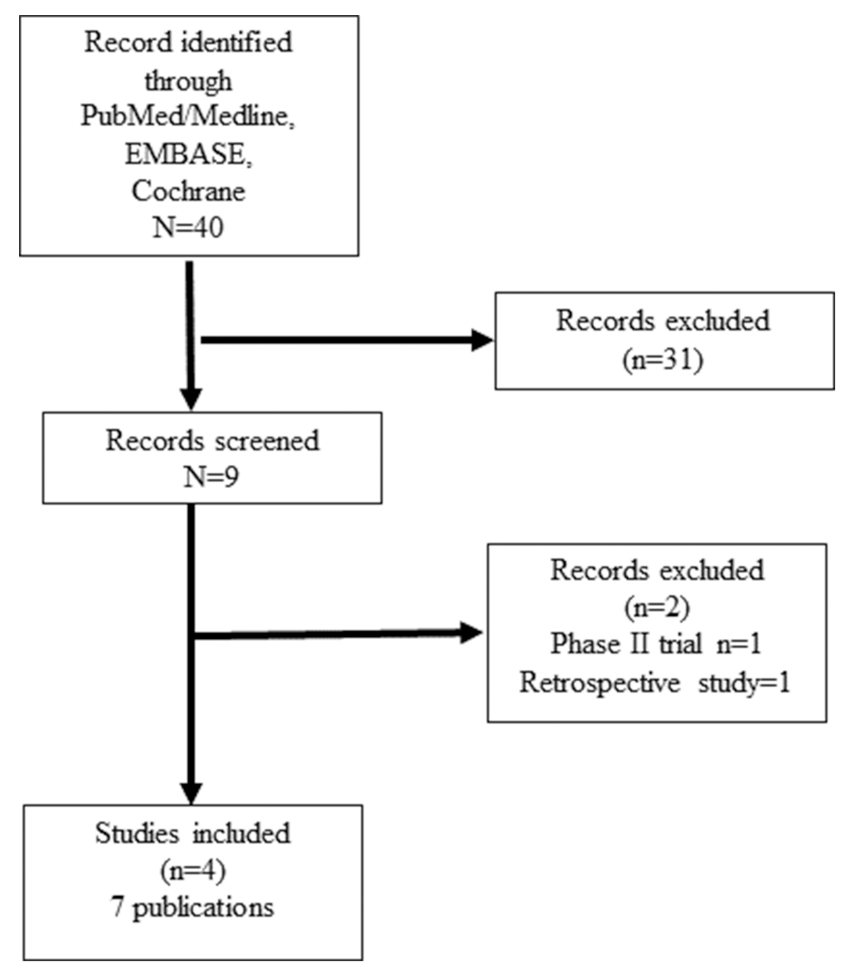

Figure I PRISMA diagram of clinical trials of ruxolitinib in polycythemia vera. 
Table I Common Instruments Used In Myeloproliferative Neoplasms For Quantitatively Measuring Symptoms And Quality Of Life

\begin{tabular}{|c|c|c|c|c|}
\hline $\begin{array}{l}\text { Abbreviated } \\
\text { QoL } \\
\text { Instrument }\end{array}$ & Full title & Scoring & Description & References \\
\hline \multirow[t]{3}{*}{ MPN-SAF } & \multirow{3}{*}{$\begin{array}{l}\text { Myeloproliferative Neoplasm } \\
\text { Symptoms Assessment Form }\end{array}$} & \multirow{3}{*}{$\begin{array}{l}\text { I4 individual symptoms scores, } \\
\text { multiplied by } 10 \text { to achieve } 0-100 \\
\text { scale. }\end{array}$} & \multirow{3}{*}{$\begin{array}{l}3 \text { symptom clusters: Mechanisms } \\
\text { related to cytokines (TSS-C); } \\
\text { Hyperviscosity (TSS-H), and } \\
\text { splenomegaly (TSS-S); MPN SAF is } \\
\text { validated in PV. }\end{array}$} & Emanuel et $\mathrm{al}^{28}$ \\
\hline & & & & Johansson et $\mathrm{al}^{33}$ \\
\hline & & & & Scherber et $\mathrm{al}^{24}$ \\
\hline PSIS & $\begin{array}{l}\text { Pruritus Symptom Impact } \\
\text { Scale }\end{array}$ & $\begin{array}{l}\text { Five point questionnaire. } 10 \text { point } \\
\text { scale; zero equals "no itching/not } \\
\text { bothered at all" and } 10 \text { equals } \\
\text { "bothered as bad as you can } \\
\text { imagine/interfered as bad as you } \\
\text { can." }\end{array}$ & $\begin{array}{l}\text { Evaluates severity, interruption of } \\
\text { daily life and improvement or } \\
\text { worsening of their itching/pruritus } \\
\text { since the start of treatment. }\end{array}$ & Vannucchi et $\mathrm{al}^{4}$ \\
\hline $\begin{array}{l}\text { EORTC-QLQ- } \\
\text { C30 }\end{array}$ & $\begin{array}{l}\text { European Organization for } \\
\text { Research and Treatment of } \\
\text { Cancer Quality of Life Core } \\
\text { Questionnaire-for all cancer } \\
\text { patients. }\end{array}$ & $\begin{array}{l}\text { Multi-item scales and single-item } \\
\text { measures. } \\
\text { Five functional scales, three } \\
\text { symptom scales, a global health } \\
\text { status/QoL scale, and six single } \\
\text { items. All of the scales and single- } \\
\text { item measures range in score from } \\
0 \text { tol } 00 \text {. }\end{array}$ & $\begin{array}{l}\text { High score represents a high/ } \\
\text { healthy status/QoL. High score for } \\
\text { a symptom represents a high level } \\
\text { of symptomatology. } \\
\text { Validated for all cancers but does } \\
\text { not capture some hematological } \\
\text { symptoms. }\end{array}$ & Aaronson et $\mathrm{al}^{34}$ \\
\hline EQ-5D-5L & $\begin{array}{l}\text { EuroQOL Group } \\
\text { non-disease specific QoL } \\
\text { instrument }\end{array}$ & $\begin{array}{l}\text { Measures health outcomes for a } \\
\text { wide range of health conditions and } \\
\text { treatments. } \\
\text { Descriptive system and a visual } \\
\text { analogue scale (EQ VAS). Five } \\
\text { dimensions: mobility, self-care, } \\
\text { usual activities, pain/discomfort, } \\
\text { and anxiety/depression. }\end{array}$ & $\begin{array}{l}\text { Endpoints are labeled "best } \\
\text { imaginable health state" and "worst } \\
\text { imaginable health state." } \\
\text { Scores can be summarized into a } \\
\text { single index score that provides a } \\
\text { simple measure of health for } \\
\text { clinical and economic appraisal. }\end{array}$ & Herdman et $\mathrm{al}^{35}$ \\
\hline WPAI & $\begin{array}{l}\text { Work Productivity and } \\
\text { Activity Impariment } \\
\text { Questionnaire }\end{array}$ & $\begin{array}{l}\text { Six-item questionnaire that } \\
\text { measures self-reported } \\
\text { productivity loss during the past } \\
\text { seven days }\end{array}$ & $\begin{array}{l}\text { Questions about absence from } \\
\text { work, hours spent at work, } \\
\text { reduction in productivity at work, } \\
\text { and reduction in productivity while } \\
\text { performing regular activities. }\end{array}$ & Relly et $\mathrm{a}^{36}$ \\
\hline PGIC & $\begin{array}{l}\text { Patient Global Impression of } \\
\text { Change }\end{array}$ & $\begin{array}{l}\text { Scale from I to } 7 \text { from "very much } \\
\text { improved" to "very much worse" }\end{array}$ & $\begin{array}{l}\text { Evaluates all aspects of patients' } \\
\text { health and assesses in there has } \\
\text { been an improvement or decline in } \\
\text { clinical status }\end{array}$ & Dorwkin et $\mathrm{al}^{37}$ \\
\hline
\end{tabular}

\section{Ethical Considerations}

This study evaluated the published data and did not require institutional ethics board approval.

\section{Results}

The initial search in PubMed, Medline, EMBASE, and Cochrane resulted in 40 scientific publications. Nine records were eligible for review. Two files were excluded because one study was retrospective, and another was a Phase 2 study. (Figure 1)

Three Phase 3 multicenter randomized trials of RUX in PV included QoL as a primary or secondary endpoint. The RESPONSE trial evaluated RUX $(n=110)$ versus standard therapy (SOC) $(n=112)$ in patients with PV and splenomegaly who were resistant or intolerant to HA. ${ }^{4}$ The primary endpoints of the study were hematocrit 
control and spleen volume; symptom reduction, QoL and safety were secondary endpoints, with PRO analysis in the same patient cohort. Patients crossed over to RUX at 32 weeks. $^{11}$

The RESPONSE 2 evaluated RUX $(\mathrm{n}=74)$ versus BAT ( $\mathrm{n}$ $=75$ ) in patients with PV without splenomegaly who were resistant to or intolerant of $\mathrm{HA}^{22}$ The primary endpoint was hematocrit control. Secondary endpoints were symptom reduction, quality of life (QoL), and safety. Patients crossed over to RUX at 28 weeks if the primary endpoint was not met.

The RELIEF study evaluated RUX ( $n=54)$ versus HA $(n=56)$ in patients who had achieved disease control with a stable dose of HA but who continued to experience PVrelated symptoms and impairment in QoL. ${ }^{23}$ The primary endpoint was a $50 \%$ improvement from baseline in myeloproliferative neoplasm related symptom assessment, the total symptom score and the cytokine symptom cluster at Week 16. Patients could cross over at 16 weeks. (Table 2)

\section{Quality Of Life And PRO Instruments}

We measured and reported the outcomes of these studies using standard instruments. Six different QoL and PRO instruments were applied: MPN-SAF, PSIS, EORTCQLQ-C30, EQ-5D-5L, WPAI, and PGIC were applied. (Table 1) MPN-SAF is the only instrument for the assessment of the prevalence and symptoms of MPNs. ${ }^{24}$

\section{The Methodological Quality Of Studies}

The overall quality score was $100 \%$ (4 out of 4 criteria met) in the three studies, as shown in Table 2. One study was missing more than $20 \%$ of PRO data, and another had more than a $20 \%$ dropout rate.

The RESPONSE trial did not consider missing patient assessments for the primary endpoint but did not mention strategies for handling missing data. PRO instruments reported an improvement of symptoms in the RUX arm, although not powered for statistical comparisons. The study did not prevent potential patient treatment bias and reported outcomes in less than $80 \%$ of patients.

RESPONSE 2 trial had a trial profile describing the number of eligible and randomized patients. PRO studies reported symptom improvements on RUX, but the follow up was short and not statistically comparable.

RELIEF study was a randomized, double-blind, doubledummy study. At the time of randomization, the discontinuation rate was $13 \%$ in RUX versus $10.7 \%$ in the HA arm. At 16 weeks, only $64.8 \%$ continue RUX versus $64.3 \%$ HA, leading to a higher than $20 \%$ dropout rate. PRO instruments showed a trend toward symptom improvement in the RUX arm that was not statistically significant. In addition to the limited number of cases, the follow-up time was only for 16 weeks, and there was a higher proportion of female patients in the HA arm.

\section{Effect Of Ruxolitinib On Patient- Reported Outcomes Symptom Control}

The primary symptoms evaluated at baseline in these studies were fatigue, insomnia, pain, dyspnea, pruritus, myalgias, night sweats, and sweats while awake (cytokine cluster), visual disturbance, dizziness, concentration difficulties, headache, numbness/tingling in hands or feet, tinnitus, skin redness (hyperviscosity cluster), abdominal discomfort and early satiety (splenomegaly symptom cluster).

In the RESPONSE trial, patients in the RUX arm reported significant symptom improvement compared to SOC at week 32 . $49 \%(36 / 74)$ of patients on RUX reported more than $50 \%$ improvement from baseline in MPN-SAF total symptom score at week 32 , versus $5 \%(4 / 81)$ in the standard treatment arm, $4 \%(2 / 49)$ with HA and 6\% (2/32) in the non-HA patients. In the cytokine cluster, symptom improvement was achieved in $64 \%$ (47/74) on RUX, versus $11 \%(9 / 80)$ on SOC, $4 \%(2 / 48)$ on $\mathrm{HA}$, and $22 \%(7 / 32)$ on non-HA respectively. In the hyperviscosity cluster, $37 \%(26 / 71)$ on (RUX, versus $13 \%(10 / 80)$ on standard treatment, $12 \%(6 / 49)$ on HA, and $13 \%(4 / 31)$ on non-HA). In the splenomegaly cluster, $62 \%$ (39/63) on RUX, versus $17 \%(12 / 71)$ on SOC, $14 \%(6 / 44)$ on HA, $22 \%(6 / 27)$ on non-HA. Patients randomized to the RUX arm also showed rapid improvements in all five components of the PSIS compared to SOC.

In RESPONSE 2, patients randomized to the RUX arm reported improvements in all individual symptoms with RUX compared to BAT where most symptoms worsened at week 28. At week 80, patients randomized initially to RUX showed an increase in the majority of individual symptom scores, except abdominal discomfort and fever. At week 28, 45.3\% $(29 / 64)$ of patients in the RUX group had a 50\% improvement in MPN-SAF TSS vs $22.7 \%(5 / 22)$ on BAT. The median percentage change from baseline in MPN-SAF TSS was $-45.3 \%$ in the RUX group versus $2.4 \%$ in the BAT group at week 28, where negative scores indicate improvement. MPNSAF TSS of at least 20 at baseline, $17(50 \%)$ of 34 patients treated with RUX achieved complete resolution of diseaserelated symptoms compared with two $(8 \%)$ of 26 patients treated with BAT. At week $80,45 \%$ of patients randomized to RUX demonstrated a higher than $50 \%$ reduction in the 


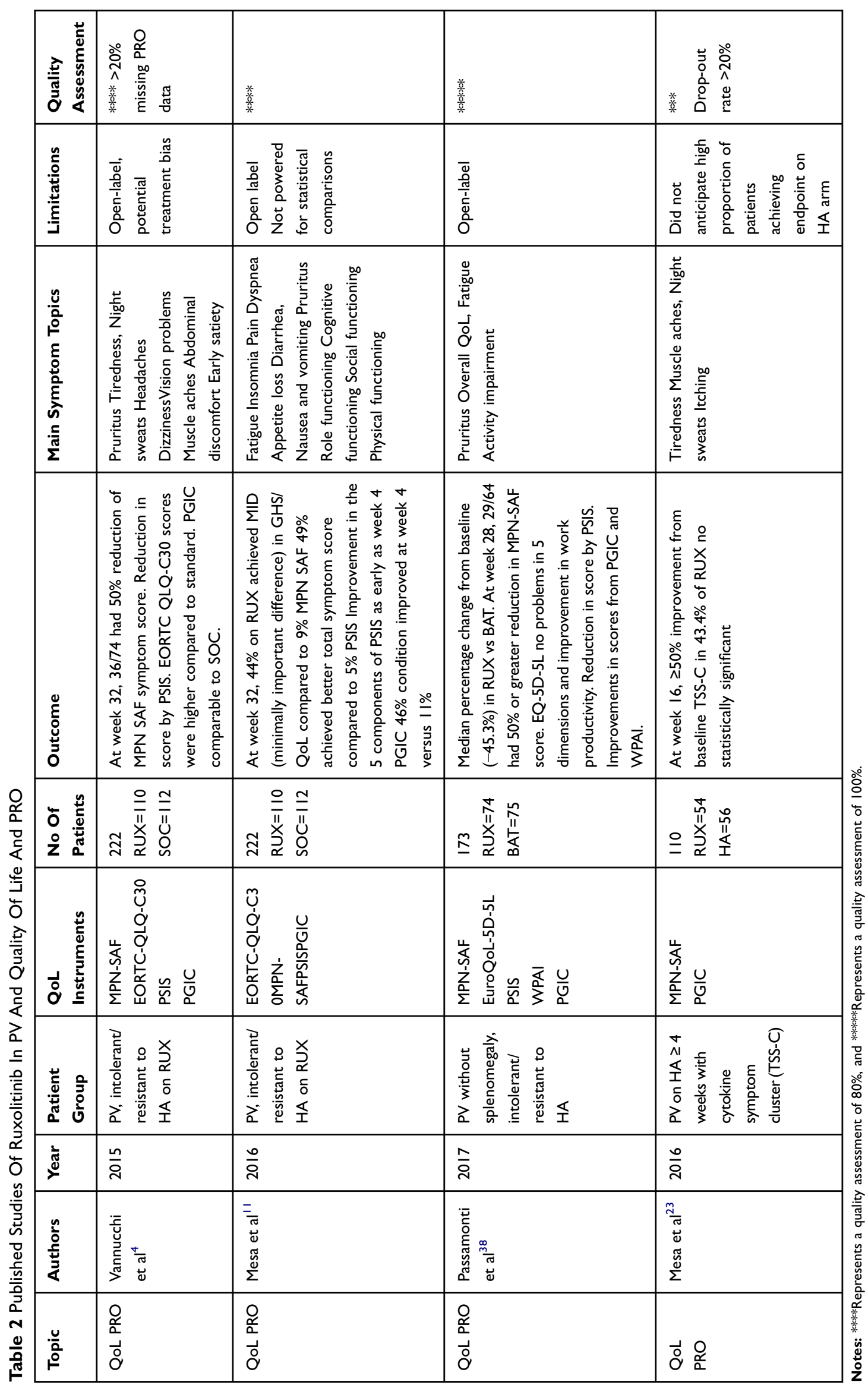


MPN-SAF TSS. It was also reflected in the sustained improvement (indicated by a negative mean change) in the total symptom scores observed in the RUX arm, with a mean (standard deviation) change of -9.0 (13.52) at week 80, consistent with what was observed at week 28 (- 10.46 [14.25]). PSIS recorded rapid improvements in the severity of pruritus in patients treated with RUX at week 28 compared to patients receiving BAT, who had a worsening in pruritus symptom severity at most assessments. At week $80,71 \%$ of patients treated with RUX showed improvement in pruritus.

In the RELIEF trial, the proportion of patients who achieved $\mathrm{a} \geq 50 \%$ improvement in the MPN-SAF TSS was similar in both groups. In the RUX arm, there was a more significant proportion of patients who achieved higher than 50\% improvement from baseline at Week 16 (RUX- 43.4\% vs HC - 29 6\%; however, the difference was not statistically significant (OR, 1 82; 95\% CI, 0 82-4 04; $\mathrm{P}=0$ 139)). A more substantial proportion of patients in the RUX arm achieved higher than 50\% improvement from baseline in the individual TSS-C symptoms compared with the HA arm, and only the difference in itchiness was statistically significant (OR, 251; 95\% CI, 110-571; $\mathrm{P}=0.027)$. The median percentage change from baseline symptoms was in favor of RUX at Week 16 and showed continued benefit at week 24 weeks and 48 weeks. The proportion of patients who achieved more than $50 \%$ improvement in the individual hyperviscosity or splenomegaly-related symptoms was similar between the treatment arms. The patients had no splenomegaly ( 0 in both the groups) and had well-controlled hematocrit (mean hematocrit in the RUX arm - $42.1 \%$ vs HA $43.7 \%$ ).

\section{Work Productivity And Activity Impairment}

In RESPONSE 2, utilizing the WPAI scale, patients reported improvement in work impairment, productivity, and days missed due to symptoms compared to the BAT where they experienced worsening of the same.

\section{Adverse Events Potentially Affecting QoL}

The most common adverse events (AE) associated with RUX were a headache (9-16\%), fatigue (7-20\%), dizziness, diarrhea, constipation, pruritus, and weight increase. An increased incidence of Herpes zoster infection occurred in patients on the RUX arm (7 (6.4\%) vs 0 in the RESPONSE trial, $1(1 \%)$ vs 0 RESPONSE- 2 trial, $1(0.5 \%)$ vs 0 in RELIEF trial). Overall, the infection rate was also slightly higher in the RUX arm. In the RESPONSE trial, the rate of infections of any grade was $41.8 \%$ in the RUX group and
$36.9 \%$ in the standard therapy group. In RESPONSE-2, 19 $(25.6 \%)$ patients had an infection of any grade (cystitis, influenza, nasopharyngitis, respiratory infection) compared to $15(20 \%)$ patients (influenza, nasopharyngitis, respiratory infection) on BAT. Four patients in the RUX group and two patients in the standard therapy group had newly diagnosed non-melanoma skin cancer (basal cell or squamous-cell carcinoma) in the RESPONSE trial; all patients but one on standard treatment had a history of skin cancer. Two patients in the RELIEF trial developed squamous cell carcinoma while on RUX. Finally, patients on RUX reported mild elevations of total cholesterol and triglycerides.

Hematological AE was mostly graded 1-2 in all three studies. Hematologic laboratory abnormalities primarily consisted of mild anemia and thrombocytopenia with RUX. Anemia and thrombocytopenia of any category were less common in the RESPONSE-2 trial (14\% and 3\% respectively) compared to the RESPONSE (43.6\% and $24.5 \%$ respectively) and RELIEF trials (37\% and $9 \%$ respectively) trials. Thromboembolic events occurred in one patient in the RUX group versus six patients in the SOC group. In the RELIEF trial, thromboembolic events occurred in two patients on the RUX arm and in two patients on the HA arm. In the RESPONSE trial, three patients on RUX progressed to myelofibrosis, and one patient received a diagnosis of AML at day 56 after randomization. One patient in the SOC arm evolved to myelofibrosis on day 101. Also, two patients assigned to standard treatment received a diagnosis of myelofibrosis on days 308 and 378 after crossover, and one progressed to AML. In RELIEF, one patient in the RUX arm developed MF transformation (on day 211, which was 24 days after the final dose of RUX) and AML (on day 216, or 29 days after the last RUX treatment).

\section{Effect Of Ruxolitinib On QoL In PV}

In the RESPONSE trial, according to the PGIC scale patients on the RUX arm (67\%) reported that their condition was "much improved" or "very much improved" at Week 32 compared to the $13 \%$ on standard therapy.

On the EORTC QLQ-C30 scale, at Week 32 patients who received RUX experienced improvements from baseline compared to significant worsening in the SOC arm. Patients who received RUX showed improvement in social, physical, role, and cognitive functional scales from baseline at Week 32, whereas patients who received standard therapy experienced a worsening of these measurements. In the emotional functioning subscale, the RUX group had a higher proportion of patients who showed improvement. A more significant 
Proportion of patients achieving a

$\geq 50 \%$ reduction in the MPN-SAF TSS

(RELIEF reported only TSS-C)

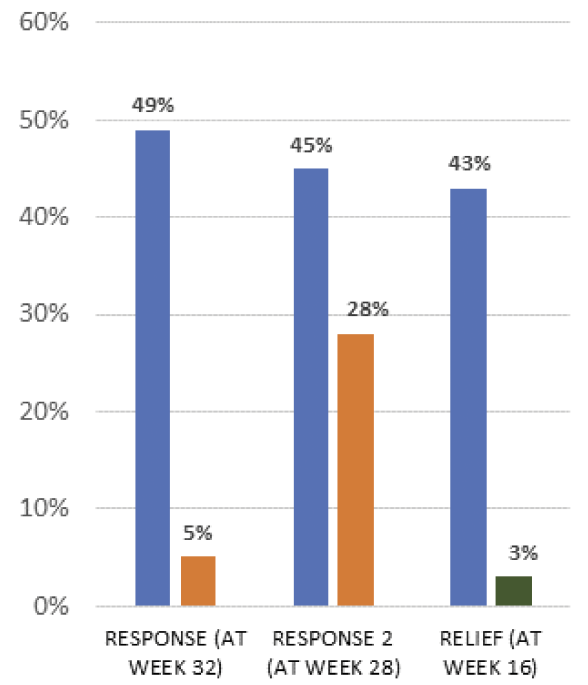

Proportion of patients who reported "very much improved" or "much improved" on PGIC scale

$$
\text { Ruxolitinib } \square \text { Standard Therapy } \square \text { Hydroxyurea }
$$

Figure 2 Histograms comparing ruxolitinib with standard therapy or hydroxyurea alone in RESPONSE at 32 weeks, RESPONSE at 28 weeks and RELIEF at 16 weeks. Graph I depicts proportion of patients who achieved a $\geq 50 \%$ reduction in the MPN-SAF TSS. The RELIEF study only reported the TSS- C (Cytokine symptoms). Graph 2 depicts the proportion of patients who reported "very much" or "much improved" on the PGIC scale.

Abbreviations: TSS, Total Symptom Score; PGIC, Patient Global Impression of Change.

proportion of patients in the RUX arm compared with the standard therapy arm (44\% vs $9 \%$ respectively) achieved a minimally significant difference (MID; $\geq a 10$-point improvement from baseline) in global health status/QoL from baseline at each post-baseline study visit through Week 32 .

In the RESPONSE-2 trial, according to the PGIC scale patients on the RUX arm, $83 \%$ reported that their condition was "much improved" or "very much improved" at Week 32 compared to $14 \%$ on standard therapy.

At Week 80 PGIC scores remained similar in patients originally randomized to RUX. On EQ-5D-5L, a higher proportion of patients in the RUX group reported having no problems in all five dimensions (mobility $52.7 \%$ vs $18.7 \%$, self-care $74.3 \%$ vs $29.3 \%$, usual activities $59.5 \%$ vs $17.3 \%$, pain/discomfort $52.7 \%$ vs $12 \%$, anxiety/depression $56.8 \%$ vs 18.7\%). EQ-5D-5L scores did not change at 80 weeks of RUX.

In the RELIEF trial, $48.1 \%$ of patients in the RUX arm reported that their PV-related symptoms were "very much improved" or "much improved" at week 16 compared to $30.4 \%$ in HA arm. (OR, 2.13; 95\% CI, 0.98-4.65). Patients in the HA arm were more likely to describe their symptoms as "minimally improved" or "no change" (HA, 55.4\%; RUX, $33.3 \%$ ) (Figure 2).

\section{Correlation Of PRO With JAK2 Allele Burden}

The RESPONSE trial evaluated the JAK2 allele burden. At week 32 , there was a $-12.2 \%$ mean change compared to a baseline (mean difference of $-34.7 \%$ ). The interpretation of this finding is limited, given that the allele burden in the study was used for exploratory analysis and not for a specific biomarker-related hypothesis. ${ }^{4}$

\section{Discussion}

Three RCTs have evaluated the impact of RUX on QoL and PRO in adult patients with PV. Although symptom burden is a very well recognized feature of MPNs, the first publication of QoL and PRO in PV was not reported until $2007 .{ }^{25}$ Since then, multiple efforts have been made to develop instruments to assess symptoms and functionality in patients with MPNs. ${ }^{24,26,27}$

The MPN-SAF is the instrument validated for QoL and PRO in myeloproliferative neoplasms. Originally consisting of a 20-item tool, the instrument subsequently incorporated 27-item tools and evaluates ten symptoms. (MPN-SAF TSS; MPN-10) ${ }^{28}$ This instrument is part of the regular assessment of MPNs (NCCN guidelines V1.2019). 
In PV, constitutively activated JAK2 recruits signal transducers and activators of transcription (STATs) to cytokine receptors and hematopoietic factors leading to chronic inflammation. ${ }^{29}$ Symptoms such as fatigue, early satiety, night sweats, and itching belong to the cluster of "cytokine symptoms" on MPN-SAF. ${ }^{24}$

Ruxolitinib is a potent kinase inhibitor of the JAK1/2STAT pathways leading to decreased expression of cytokines and cell growth factors necessary for hematopoiesis. ${ }^{18,30}$ Other JAK2 inhibitors share similar mechanisms of action and are under evaluation in other myeloproliferative neoplasms.

In myelofibrosis, RUX is effective in reducing spleen size and significantly improving the total symptom score according to the modified Myelofibrosis Symptom Assessment Form (MFSAF) ${ }^{31}$ More recently, fedratinib, a selective JAK2 inhibitor, received FDA approval for the management of primary or secondary myelofibrosis. ${ }^{32}$ Although fedratinib improved symptom responses per TSS from baseline up to 24 weeks, ${ }^{33}$ no ongoing studies are evaluating its efficacy in PV.

An open-label, randomized Phase 2 study evaluated momelotinib, a JAK1/JAK2 inhibitor in PV; ${ }^{34}$ the study was terminated because of limited efficacy; momelotinib did not change symptom burden per MPN-SAF TSS.
RESPONSE and RESPONSE 2 demonstrated the efficacy of RUX in decreasing symptom burden in PV. (Table 1) The authors proposed that the results from the studies have real-life applications since the comparison group was the best available therapy. However, an important caveat is that the open-label design of RESPONSE and RESPONSE 2 could not etermine statistical differences in PROs between arms. Additionally, the evaluation of PRO in PV patients treated with RUX, who do not have splenomegaly, will require further study. The effect of RUX on PRO needs to consider other variables such as age, gender, and leukemic transformation. The role of PROs as a prognostic marker in PV and their correlation with cytokine activity are also exciting options to consider for further analyses.

Conversely, RELIEF did not show statistical differences in PRO in the RUX arm compared to HA. ${ }^{23}$ Despite the design limitations of the three studies, the current data strongly supports the efficacy of RUX in PRO compared to other conventional PV therapies (Figure 1).

The progressive incorporation of PROs in RCTs in oncology is ongoing. ${ }^{35}$ Current clinical trials in PV include PRO data in the efficacy assessment of MPNs (Table 3).

Table 3 Ongoing Clinical Trials In Polycythemia Vera Incorporating PRO In Efficacy Assessment

\begin{tabular}{|c|c|c|c|c|}
\hline Title & $\begin{array}{l}\text { Clinical Trial } \\
\text { Identifier }\end{array}$ & PRO Instruments & Phase & $\begin{array}{l}\text { Recruitment } \\
\text { Status }\end{array}$ \\
\hline $\begin{array}{l}\text { TGR-I202 + Ruxolitinib in Subjects With Myelofibrosis, } \\
\text { MDS/MPN, or Polycythemia Vera Resistant to } \\
\text { Hydroxyurea }\end{array}$ & NCT02493530 & MPN-SAF TSS & Phase I & Recruiting \\
\hline $\begin{array}{l}\text { Expanded Treatment Protocol (ETP) of Ruxolitinib in } \\
\text { Patients With Polycythemia Vera Who Are Hydroxyurea } \\
\text { Resistant or Intolerant and for Whom no Treatment } \\
\text { Alternatives Are Available. }\end{array}$ & NCT02292446 & MPN-SAF & Phase III & Completed \\
\hline $\begin{array}{l}\text { SAR302503 in Patients With Polycythemia Vera or } \\
\text { Essential Thrombocythemia }\end{array}$ & NCTOI420783 & MPN-SAF & Phase II & Completed \\
\hline $\begin{array}{l}\text { Low Dose Interferon Alpha Versus Hydroxyurea in } \\
\text { Treatment of Chronic Myeloid Neoplasms (DALIAH) }\end{array}$ & NСT0I387763 & $\begin{array}{l}\text { EORTC QLQ C-30 and MPN- } \\
\text { SAF }\end{array}$ & Phase III & $\begin{array}{l}\text { Active, not } \\
\text { recruiting }\end{array}$ \\
\hline $\begin{array}{l}\text { The Benefit/Risk Profile of AOP20I4 in Low-risk Patients } \\
\text { With PV (Low-PV) }\end{array}$ & NCT03003325 & $\begin{array}{l}\text { Functional Assessment of cancer } \\
\text { Therapy-Anaemia (FACT-An) } \\
\text { and MPN-SAF TSS }\end{array}$ & Phase II & Recruiting \\
\hline $\begin{array}{l}\text { Idasanutlin Monotherapy in Patients With Hydroxyurea- } \\
\text { Resistant/Intolerant Polycythemia Vera }\end{array}$ & NCT03287245 & $\begin{array}{l}\text { MPN-SAF TSS } \\
\text { EORTC QLQ-C } 30 \\
\text { PGIC }\end{array}$ & Phase II & Recruiting \\
\hline $\begin{array}{l}\text { KRT-232 Compared to Ruxolitinib in Patients With } \\
\text { Phlebotomy-Dependent Polycythemia Vera }\end{array}$ & NCT03669965 & $\begin{array}{l}\text { MPN-SAF TSS } \\
\text { EORTC-QLQ-C30 }\end{array}$ & Phase II & Recruiting \\
\hline
\end{tabular}


The data regarding the benefit of RUX versus standard treatment in HA intolerance/resistant PV is limited. The effect of MPN treatments in PRO and QoL needs confirmation with large-scale and long-term studies. Interventions directed to improve PRO in MPNs would provide a better understanding of symptoms, optimize therapy selection, and increase patient survival.

\section{Conclusion}

RUX in PV patients who are intolerant or resistant to HA is associated with significantly decreased symptom burden. HA is an effective cytoreductive agent, but its effect PRO is primarily limited to pruritus control. PRO and QoL assessments are an integral part of the evaluation and treatment of MPNs, and patients with PV on RUX will experience improvements in PRO in addition to improving hematological parameters.

\section{Disclosure}

CAY reports involvement in advisory boards with JAZZ in 2018/2019 and with Pfizer in 2017. The authors report no other conflicts of interest in this work.

\section{References}

1. Vannucchi AM, Guglielmelli P. What are the current treatment approaches for patients with Polycythemia vera and essential thrombocythemia? Hematology. 2017;(1):480-488. doi:10.1182/asheducation-2017.1.480

2. Tefferi A. Primary myelofibrosis: 2019 update on diagnosis, riskstratification and management. Am J Hematol. 2018;93:1551-1560. doi:10.1002/ajh.25230

3. Mesa R, Boccia RV, Grunwald MR, et al. Patient-reported outcomes data from REVEAL at the Time of Enrollment (Baseline): a prospective observational study of patients with Polycythemia vera in the United States. Clin Lymphoma, Myeloma Leuk. 2018;18:590-596. doi:10.1016/j.clml.2018.05.020

4. Vannucchi AM, Kiladjian JJ, Griesshammer M, et al. Ruxolitinib versus standard therapy for the treatment of Polycythemia vera. $N$ Engl J Med. 2015;372:426-435. doi:10.1038/mt.2009.265

5. Mesa R, Miller CB, Thyne M, et al. Myeloproliferative neoplasms (MPNs) have a significant impact on patients' overall health and productivity: the MPN Landmark survey. BMC Cancer. 2016;16:167. doi:10.1186/s12885-016-2208-2

6. Abelsson J, Andréasson B, Samuelsson J, et al. Patients with polycythemia vera have worst impairment of quality of life among patients with newly diagnosed myeloproliferative neoplasms. Leuk Lymphoma. 2013;54(10):2226-2230.

7. Stein BL, Moliterno AR, Tiu RV. Polycythemia vera disease burden: contributing factors, impact on quality of life, and emerging treatment options. Ann Hematol. 2014;93:1965-1976. doi:10.1007/s00277-0142205-y

8. Nelson EC, Eftimovska E, Lind C, et al. Patient reported outcome measures in practice. BMJ. 2015;350:g7818-g7818. doi:10.1136/bmj. g7818
9. Buckley SA, Kirtane K, Walter RB, et al. Patient-reported outcomes in acute myeloid leukemia: where are we now? Blood Rev. 2018;32:81-87. doi:10.1016/j.blre.2017.08.010

10. Bryant AL, Drier SW, Lee S, Bennett AV. A systematic review of patient reported outcomes in phase II or III clinical trials of myelodysplastic syndromes and acute myeloid leukemia. Leuk Res. 2018;70:106-116. doi:10.1016/j.leukres.2018.06.006

11. Mesa R, Verstovsek S, Kiladjian JJ, et al. Changes in quality of life and disease-related symptoms in patients with polycythemia vera receiving ruxolitinib or standard therapy. Eur J Haematol. 2016;97:192-200. doi:10.1111/ejh.12707

12. Blum S, Martins F, Alberio L. Ruxolitinib in the treatment of polycythemia vera: patient selection and special considerations. J Blood Med. 2016;7:205-215. doi:10.2147/JBM.S102471

13. Mesa RA, Miller CB, Mascarenhas JO, et al. Hydroxyurea treatment history and quality of life in patients with polycythemia vera: results from the MPN landmark survey in the United States. Blood. 2015;126 (23):4077.

14. Scherber R, Dueck A, Kiladjian J, et al. Conventional therapeutic options have limited impact on MPN symptoms: insights from a prospective analysis of the MPN-SAF TSS. Haematologica. 2012.

15. Alvarez-Larrán A, Pereira A, Cervantes F, et al. Assessment and prognostic value of the European leukemiaNet criteria for clinicohematologic response, resistance, and intolerance to hydroxyurea in polycythemia vera. Blood. 2012;119:1363-1369. doi:10.1182/blood2011-10-387787

16. Raedler LA. Jakafi (Ruxolitinib): first FDA-approved medication for the treatment of patients with Polycythemia vera. Am Heal Drug Benefits. 2015;8:75-79.

17. Barosi G, Mesa R, Finazzi G, et al. Revised response criteria for polycythemia vera and essential thrombocythemia: an ELN and IWG-MRT consensus project. Blood. 2013;121(23):4778-4781. doi:10.1182/blood2013-01-478891

18. Verstovsek S, Mesa RA, Gotlib J, et al. A double-blind, placebocontrolled trial of ruxolitinib for myelofibrosis. $N$ Engl J Med. 2012;366(9):799-807. doi:10.1056/NEJMoa1110557

19. Harrison C, Kiladjian J, Al-Ali HK, et al. JAK inhibition with ruxolitinib versus best available therapy for myelofibrosis. $N$ Engl J Med. 2012;366(9):787-798.

20. Moher D, Liberati A, Tetzlaff J, Altman D. Preferred reporting items for systematic reviews and metaanalyses: the PRISMA statement. PLoS Med. 2009;6(6):e1000097. doi:10.1371/journal. pmed1

21. Hong QN, Gonzalez-Reyes A, Pluye P. Improving the usefulness of a tool for appraising the quality of qualitative, quantitative and mixed methods studies, the Mixed Methods Appraisal Tool (MMAT). J Eval Clin Pract. 2018;24(3):459-467. doi:10.1111/jep.12884

22. P F, G M, P F, et al. Ruxolitinib for the treatment of inadequately controlled polycythaemia vera without splenomegaly (RESPONSE2): a randomised, open-label, phase 3b study. Lancet Oncol. 2017;18 (1):p. 88-99. doi:10.1016/S1470-2045(16)30558-7

23. Mesa R, Vannucchi AM, Yacoub A, et al. The efficacy and safety of continued hydroxycarbamide therapy versus switching to ruxolitinib in patients with polycythaemia vera: a randomized, double-blind, double-dummy, symptom study (RELIEF). Br J Haematol. 2017;176(1):76-85. doi:10.1111/bjh.14382

24. Scherber R, Dueck AC, Johansson P, et al. The Myeloproliferative Neoplasm Symptom Assessment Form (MPN-SAF): international prospective validation and reliability trial in 402 patients. Blood. 2011;118(2):401-408. doi:10.1182/blood-2011-01-328955

25. Mesa RA, Niblack J, Wadleigh M, et al. The burden of fatigue and quality of life in myeloproliferative disorders (MPDs): an international internet-based survey of 1179 MPD patients. Cancer. 2007;109 (1):68-76. doi:10.1002/cncr.22365 
26. Mesa RA, Schwager S, Radia D, et al. The Myelofibrosis Symptom Assessment Form (MFSAF): an evidence-based brief inventory to measure quality of life and symptomatic response to treatment in myelofibrosis. Leuk Res. 2009;33(9):1199-1203. doi:10.1016/j. leukres.2009.01.035

27. Mesa RA, Gotlib J, Gupta V, et al. Effect of ruxolitinib therapy on myelofibrosis-related symptoms and other patient-reported outcomes in COMFORT-I: a randomized, double-blind, placebo-controlled trial. J Clin Oncol. 2013;31(10):1285-1292. doi:10.1200/ JCO.2012.44.4489

28. Emanuel RM, Dueck AC, Geyer HL, et al. Myeloproliferative neoplasm (MPN) symptom assessment form total symptom score: prospective international assessment of an abbreviated symptom burden scoring system among patients with MPNs. J Clin Oncol. 2012;30(33):4098-4103. doi:10.1200/JCO.2012.42.3863

29. Pourcelot E, Trocme C, Mondet J, et al. Cytokine profiles in polycythemia vera and essential thrombocythemia patients: clinical implications. Exp Hematol. 2014;42(5):360-368. doi:10.1016/j.exphem.2 014.01 .006

30. Verstovsek S, Kantarjian H, Mesa RA, et al. Safety and Efficacy of INCB018424, a JAK1 and JAK2 Inhibitor, in Myelofibrosis. $N$ Engl J Med. 2010;363(12):1117-1127. doi:10.1056/NEJMoa 1002028
31. Mesa RA, Kantarjian H, Tefferi A, et al. Evaluating the serial use of the myelofibrosis symptom assessment form for measuring symptomatic improvement: performance in 87 myelofibrosis patients on a JAK1 and JAK2 inhibitor (INCB018424) clinical trial. Cancer. 2011;117(21):4869-4877. doi:10.1002/cncr.26129

32. Fedratinib becomes new option in myelofibrosis. Cancer Discov. 2019;9(10):1332. doi:10.1158/2159-8290.CD-NB2019-102

33. Pardanani A, Harrison C, Cortes JE, et al. Safety and efficacy of fedratinib in patients with primary or secondary myelofibrosis: A randomized clinical trial. JAMA Oncol. 2015;1(5):643-51. doi:10.1001/jamaoncol.2015.1590

34. Verstovsek S, Courby S, Griesshammer M, et al. A phase 2 study of momelotinib, a potent JAK1 and JAK2 inhibitor, in patients with polycythemia vera or essential thrombocythemia. Leuk Res. 2017. doi:10.1016/j.leukres.2017.05.002

35. Trotti A, Colevas AD, Setser A, Basch E. Patient-reported outcomes and the evolution of adverse event reporting in oncology. $J$ Clin Oncol. 2007;25(32):5121-5127.
Journal of Blood Medicine

\section{Publish your work in this journal}

The Journal of Blood Medicine is an international, peer-reviewed, open access, online journal publishing laboratory, experimental and clinical aspects of all aspect pertaining to blood based medicine including but not limited to: Transfusion Medicine; Blood collection, Donor issues, Transmittable diseases, and Blood banking logistics; Immunohematology; Artificial and alternative blood based

\section{Dovepress}

therapeutics; Hematology; Biotechnology/nanotechnology of blood related medicine; Legal aspects of blood medicine; Historical perspectives. The manuscript management system is completely online and includes a very quick and fair peer-review system. Visit http://www.dovepress.com/testimonials.php to read real quotes from published authors. 